Año 16, Vol. 11, número 21, agosto- diciembre 2021

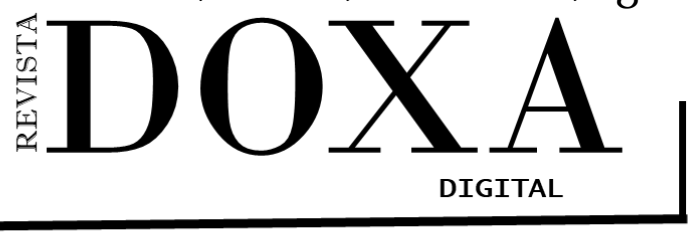

eISSN: 2594-2786
Sección: Ciencias Sociales

Recibido: Mayo 2021

Aceptado: Agosto 2021

DOI: $10.52191 /$ rdojs.2021.207

Pág. 8- 34

\title{
La inseguridad alimentaria, la malnutrición y la obesidad: desde el enfoque de las capacidades
}

\author{
Food insecurity, malnutrition and obesity: from a capacity-building approach
}

Lorena Arias Díaz

\section{RESUMEN}

La inseguridad alimentaria, la malnutrición alimenticia y la obesidad, principalmente infantil, se han convertido en tres grandes problemas de salud pública, que requieren una atención urgente e inmediata. Sin embargo, aunque existen diversos programas dirigidos a la disminución o control de dichas situaciones problemáticas, la realidad es que, no se ha logrado un impacto positivo ni en lo cuantitativo ni en lo cualitativo. Es por esta razón, que consideramos el enfoque de capacidades propuesto por Amartya Sen, como una posible alternativa, para posteriores políticas públicas y sociales, encaminadas a la compensación de ciertas incapacidades alimentarias de gran parte de la población mexicana

PALABRAS CLAVE: Desarrollo humano, capacidades, incapacidades, inseguridad alimentaria, malnutrición alimenticia

\begin{abstract}
Food insecurity, food malnutrition and obesity, mainly children, have become major public health problems, requiring urgent and immediate attention. However, although there are a number of programs aimed at reducing or controlling these problematic situations, the reality is that no positive impact has been achieved in the quantitative or qualitative situations. It is for this reason that we consider the capacity approach proposed by Amartya Sen, as a possible alternative, for subsequent public and social policies, aimed at compensating for certain food incapacity of much of the Mexican population
\end{abstract}

KEYWORDS: human development, capacities, disabilities, food insecurity, food malnutrition

* Estudiante de tiempo completo del Doctorado en Ciencias Sociales del Colegio de Jalisco. Licenciada en Ciencias Políticas y Administración Pública por la Universidad Autónoma de Aguascalientes, Maestra en Gestión, Desarrollo y Gobierno por la Universidad de Guanajuato. Contacto: lorenaariasdiaz@gmail.com 
DOI: 10.52191/rdojs.2021.207 La seguridad alimentaria, la malnutrición ... Págs.: 8-34

\section{Introducción}

El objetivo de este artículo, es realizar un apartado teórico, en el cual, definiremos de forma general los conceptos de desarrollo humano, la capacidad y la justicia, para posteriormente abordar desde estas perspectivas, problemas que influyen considerablemente en la salud pública en México como lo son la inseguridad alimentaria, la malnutrición y la obesidad. Centrándonos, principalmente en el enfoque de capacidades, propuesto por ganador del Premio Nobel de Economía en 1998, hablamos del intelectual indio Amartya Sen.

La teoría del economista originario de la India, trasciende la creencia de que el crecimiento económico, el Producto Interno Bruto (PIB) y el ingreso per cápita, significan por añadidura un incremento en el desarrollo y bienestar humano. La propuesta de Sen (2010), se encamina a lo que él nombra como "enfoque de capacidades", que puede definirse someramente como la agencia de una persona para hacer determinadas acciones y funciones, es decir, la libertad personal de elegir, por ejemplo, un determinado estilo de vida. En resumidas palabras, es la capacidad de elección libre en función a las valoraciones y los deseos de cada individuo.

Sin embargo, es un hecho innegable que la existencia de desigualdad socioeconómica entre naciones, entre regiones y entre individuos de un mismo lugar, terminan por limitar la capacidad que poseen los sujetos. Pese a la importancia de lo anterior, Sen (2000), en su teoría destaca más la privación de capacidades, oportunidades y libertades, que a la pobreza de la renta, ya que, estas vulneran en mayor medida el desarrollo humano de las personas.

En el país mexicano, existe un problema alarmante de alimentación, ya sea, por incapacidad económica o por el inacceso a alimentos saludables. La malnutrición e inseguridad alimentaria, han impactado drásticamente en los índices elevados de las prevalencias combinadas de sobrepeso y obesidad (SPyO), en todos los grupos de edad, y a su vez, dicha condición física se convierte en un factor de alto riesgo, para desarrollar enfermedades crónico-degenerativas no transmisibles (ECDNT), que merman la calidad y esperanza de vida e incapacitan a millones de mexicanos.

En próximos apartados, se dará cuenta de manera más amplia lo expuesto hasta ahora, para dicha tarea se realiza un análisis de la inseguridad alimentaria, la malnutrición y la obesidad desde la teoría de capacidades y justicia, asimismo, recuperamos datos y cifras de dichos temas, a través de fuentes oficiales de organizaciones e instituciones internacionales y nacionales.

Se divide este ensayo cuatro apartados, en el primero, se ofrece una contextualización del 
DOI: 10.52 191/rdojs.2021.207 La seguridad alimentaria, la malnutrición ... Págs.: 8-34

desarrollo humano; en el segundo, se trata de explicar el por qué considerar que la inseguridad alimentaria, la malnutrición y la obesidad, pueden convertirse en incapacidades que afectan negativamente al desarrollo humano; en el tercero se ve la dualidad de la justicia y la seguridad alimentaria, bajo las posturas de Sen y Rawls. Y finalmente, las conclusiones del trabajo.

\section{Desarrollo humano: un acercamiento teórico}

El término desarrollo tiene su origen en las ciencias naturales, específicamente en la biología, aunque posteriormente comenzó a utilizarse de forma consistente en las ciencias sociales, incluida desde luego la economía. Dicho concepto, a menudo suele causar confusión e incluso se ha utilizado como sinónimo de otras palabras, que si bien, pueden ser vinculadas con el desarrollo, no necesariamente significan lo mismo, tal es caso del llamado crecimiento económico.

De acuerdo a la página oficial de la Real Academia Española (2020), se puede entender al desarrollo como las acciones o efectos para desarrollar o desarrollarse, por ejemplo, las distintas etapas por las que transita un humano, que van desde la fecundación hasta senectud. Desde un enfoque meramente económico, se dice que es el proceso transformación de determinada economía hacia la consecución de mejores niveles de vida.

Al respecto, Preston (1999), realiza un recorrido histórico en relación a las teorías del desarrollo en los años posteriores a la Segunda Guerra Mundial (SGM), dichos postulados se diseñaron con la intención de obtener un modelo que generara mayor crecimiento económico, y que este, pudiera ser también aplicable a los países del tercer mundo o en vías de desarrollo. Se consideraba importante y trascendental, replicar los modelos de naciones desarrollas en economía e industria, y se afirmaba que, una vez llevados a la práctica, los habitantes de dichas regiones "subdesarrolladas", comenzarían a gozar niveles mayores prosperidad en todos los sentidos.

En este mismo sentido Gudynas (2012), dice que a partir de la década de los cincuenta del siglo XX, la definición de desarrollo implicaba necesariamente el crecimiento económico. Asimismo, se afirmaba que con aumento del PIB y del ingreso per cápita de determinados países y poblaciones, se garantizaría a las personas una mejor calidad de vida.

En relación al Producto Interno Bruto (PIB), es importante señalar que, si bien, el crecimiento económico de un determinado país, puede ser un factor positivo para atraer inversiones extranjeras, este no garantiza por sí solo, un desarrollo homogéneo y una mejor calidad de vida de todos sus habitantes. 
DOI: 10.52191/rdojs.2021.207 La seguridad alimentaria, la malnutrición ... Págs.: 8-34

Aunque, el propio Amartya Sen (2000), reconoce que la riqueza puede ser hasta cierto punto determinante en el desarrollo de capacidades individuales ${ }^{1}$ no es la única limitante, para sostener lo dicho expone un ejemplo en dos sentidos distintos inversos: "1) una renta baja puede ser una importante causa del analfabetismo y de merma en la salud, así como del hambre y la desnutrición, y 2), a la inversa, una mejora de la salud contribuye a obtener una renta más alta." (p. 36).

Con lo anterior, Sen trata de explicar que, en algunos casos el ingreso de la renta es importante para determinar la forma de vida de diversas personas, empero, existen otros factores que limitan o expanden ciertas capacidades y libertades básicas, como puede ser perfectamente la inseguridad alimentaria y la malnutrición o en un sentido positivo la seguridad alimentaria. Una vez, expuesto brevemente todo lo expuesto, quisiéramos centrarnos en el "nuevo paradigma de desarrollo" conocido como humano o de las capacidades.

Amartya Sen (2010), sostiene que la evaluación del desarrollo no puede, ni debe alejarse de la vida y de la libertad que las personas puedan tener, es por esta razón, que el desarrollo no debe limitarse a mediciones como el aumento del Producto Nacional Bruto (PNB), el Producto Interno Bruto, ingresos personales o el crecimiento industrial, se debe trascender y pensar en aspectos fundamentales como la calidad de vida, la educación, así como, todo lo relacionado a las libertades sustanciales de todos los individuos, tales condiciones deben ser centrales en la búsqueda del desarrollo humano.

Por lo tanto, para Sen el desarrollo es "un proceso de expansión de las libertades reales de que disfrutan los individuos." (2000, p. 19). Aunado a esto, el autor expresa que, el verdadero desarrollo demanda eliminar todas aquellas causas de privación de las libertades, como lo son la pobreza, los gobiernos tiránicos, la falta de oportunidades económicas, entre otras. Nosotros agregaríamos a la inseguridad alimentaria, como una fuente que limita el pleno de desarrollo de las capacidades, esto lo veremos más a fondo en próximos apartados.

Los derechos humanos juegan un papel primordial, si lo que, se desea es aumentar las capacidades, oportunidades y libertades de todos y cada uno de los individuos sobre la faz de la tierra.

De acuerdo a la Organización de las Naciones Unidas (ONU, 2021), la Declaración Universal de los Derechos Humanos, esta fue promulgada por la Asamblea General de las Naciones Unidas

1 Término que expondremos a detalle en el segundo apartado del ensayo. 
DOI: 10.52191/rdojs.2021.207 La seguridad alimentaria, la malnutrición ... Págs.: 8-34

en París en el año de 1948, la cual, estaba compuesta por más de 50 Estados miembros hasta ese entonces y esta fue el resultado de las lamentables consecuencias bélicas, provocadas por la SGM. Lo que se pretendía era "garantizar los derechos de todas las personas en cualquier lugar y en todo momento." (ONU, 2021, recuperado de: https://www.un.org/es/about-us/udhr/history-of-thedeclaration)

En la actualidad existe un gran catálogo o listado de derechos humanos, que en el proceso de la diplomacia los Estados miembros de las Naciones Unidas, ${ }^{2}$ se comprometen a garantizarlos, aplicarlos y respetarlos.

Algunos derechos fundamentales, de acuerdo a la Organización de las Naciones Unidas (2021) son:

- Derecho a la libertad e igualdad.

- Derecho a la vida.

- Derecho a una personalidad jurídica.

- Derecho de expresión y opinión.

- Derecho de asociación.

- Derecho a la seguridad social.

- Derecho al trabajo.

- Derecho a la educación.

- Derecho a un nivel de vida adecuado, esto incluye salud, bienestar y en especial una adecuada alimentación, así como, contar con vestidos, vivienda, asistencia médica y servicios sociales.

Como se puede observar, todos y cada uno de los derechos se relacionan y se influyen mutuamente, para este artículo nos enfocaremos en el último. Es pertinente, también enunciar que entre los diez derechos fundamentales de la niñez, según encontramos el derecho a una alimentación suficiente y adecuada.

En el caso mexicano, se encuentrz en el Artículo 4o. Constitucional, textualmente lo siguiente: "Toda persona tiene derecho a la alimentación nutritiva, suficiente y de calidad. El Estado lo garantizará." (Constitución Política de los Estados Unidos Mexicanos, 2021, p. 10) 
DOI: 10.52191/rdojs.2021.207 La seguridad alimentaria, la malnutrición ... Págs.: 8-34

Sin lugar a dudas, tanto la Derechos Humanos por parte de la ONU como la incorporación de garantías individuales y sociales dentro de la Constitución Política de los Estados Unidos Mexicanos (CPEUM) es un gran avance, lo cierto es que lo escrito en el papel difiere por mucho la situación en la que se encuentran millones de personas en México y el mundo. Por lo tanto, el desarrollo de capacidades individuales y grupales es sometido ante la situación y el contexto en el que se vive en el día a día, sin importar el crecimiento económico y los avances en la legislación, nos encontramos ante una realidad que es diametralmente a lo esperado. Es por esta razón, que vale la pena voltear la mirada en dirección a otra alternativa como es la que plantea Sen con su teoría de la justicia y el enfoque de capacidades.

En este mismo sentido, se observa lo expuesto por una de las pioneras del enfoque de las capacidades, hablamos de Nussbaum (2012), quien también realiza una crítica a los diseñadores y formuladores de políticas públicas, los cuales, a su criterio, distorsionaban la realidad humana, ya que, para ellos simplemente con el incremento del PIB de un determinado país, aumentaría automáticamente la calidad y el bienestar de vida de las personas.

[...] [E]se enfoque indiferenciado los alentaba a centrar todos sus esfuerzos en el capítulo del crecimiento económico, sin prestar atención al nivel de vida de sus habitantes más pobres y sin abordar tampoco cuestiones como la salud y la educación, que normalmente no mejoran con el crecimiento económico sin más. (Nussbaum, 2012, p. 13)

Continuando con Nussbaum (2012), asegura que el enfoque del PIB, debe ser remplazado por el enfoque de las capacidades, el cual, debe contemplar lo que realmente las personas son capaces de ser y hacer, así como, tener la capacidad de observar las oportunidades con las que cuentan, para ser o hacer. Por lo tanto, el objetivo fundamental del desarrollo "es crear un ambiente propicio para que los seres humanos disfruten de una vida prolongada, saludable y creativa." ( $p$. 21)

Otro aporte en este cambio de paradigma de desarrollo, es el propuesto por Kaushik (2002), quien expone que el desarrollo no debe centrarse exclusivamente en indicadores como Producto Interno Bruto, el Producto Interno Neto (PIN), el Producto Nacional Bruto (PNB), el Producto Nacional Neto (PNN), entre otros. Lo ideal, sería encaminarse al desarrollo humano o desarrollo ampliado, que no es otra cosa que el enfoque de capacidades de Amartya Sen.

Los intentos para darles a estas metas un enfoque definido como en la construcción del Índice de Desarrollo Humano, construido por el Programa de las Naciones Unidas para el Desarrollo (PNUD), han sido 
DOI: 10.52191/rdojs.2021.207 La seguridad alimentaria, la malnutrición ... Págs.: 8-34

inevitablemente criticados por su arbitrariedad. Aún sobre éste, sin embargo, uno puede argumentar que es mejor ser algo arbitrario pero tener amplio objetivo correcto que tener un objetivo claramente definido pero moralmente indefendible. (Kaushik, 2002, p. 49)

El mayor problema, para implementar un modelo desarrollo donde impere lo humano sobre lo económico, nos dice Kaushik (2002), es que los gobiernos nacionales han sido apáticos a las indicaciones propuestas por organismos internacionales, tal con es garantizar los derechos fundamentales. Finalmente, el autor expone algunas de las razones que justifican al modelo propuesto:

- $\quad$ El desarrollo humano es un fin deseado en sí mismo.

- $\quad$ Puede generar mayores niveles de productividad y así incrementarse el poder adquisitivo de bienes y servicios.

- Se disminuyen los niveles demográficos.

- $\quad$ Contribuye a un mejor manejo del medio ambiente.

- Puede consolidar las bases civiles y democráticas.

- Genera estabilidad política.

Para cerrar, lo relacionado a esta sección del artículo, es necesario precisar que el Índice de Desarrollo Humano (IDH), propuesto por el Programa de las Naciones Unidas para el Desarrollo (PNUD), se basó en el enfoque de capacidades de Amartya $\mathrm{Sen}^{3}$, quien además participó en su diseño, y aunque, pareciera un poco limitado, es mucho más cercano a la realidad de los países y sus habitantes.

El desarrollo de un país no puede ser entendido desde la perspectiva única del crecimiento económico. El propósito final del desarrollo se encuentra en cada uno de sus habitantes y en las posibilidades que ellos tienen para elegir una vida en la que puedan realizar a plenitud su potencial como seres humanos. (Programa de las Naciones Unidas para el Desarrollo, 2020, recuperado de: https://www.mx.undp.org/content/mexico/es/home/ourwork/ povertyreduction/in_depth/desarrollo-humano.html)

De acuerdo al PNUD México (2020), el IDH cuenta con tres componentes de medición, los cuales a saber son: índice de salud, índice de educación e índice de ingreso ${ }^{4}$. A continuación, presentemos un mapa conceptual del Índice de Desarrollo Humano.

3 "Aunque la idea de elaborar esta clase de medidas se debe al economista paquistaní Mahbub ul Haq, Sen colaboró con él en la creación de los índices a principios de los años noventa como asesor de la Organización de las Naciones Unidas [...]" (Cejudo, 2006, p. 366)

4 El Programa de las Naciones Unidas para el Desarrollo (2020), dice que "los cambios en la dimensión de ingreso buscan reflejar con mayor precisión los recursos internos del país al emplear el Ingreso Nacional Bruto (INB) per cápita 
DOI: 10.52191/rdojs.2021.207 La seguridad alimentaria, la malnutrición ... Págs.: 8-34

Figura 1. Índice de Desarrollo Humano

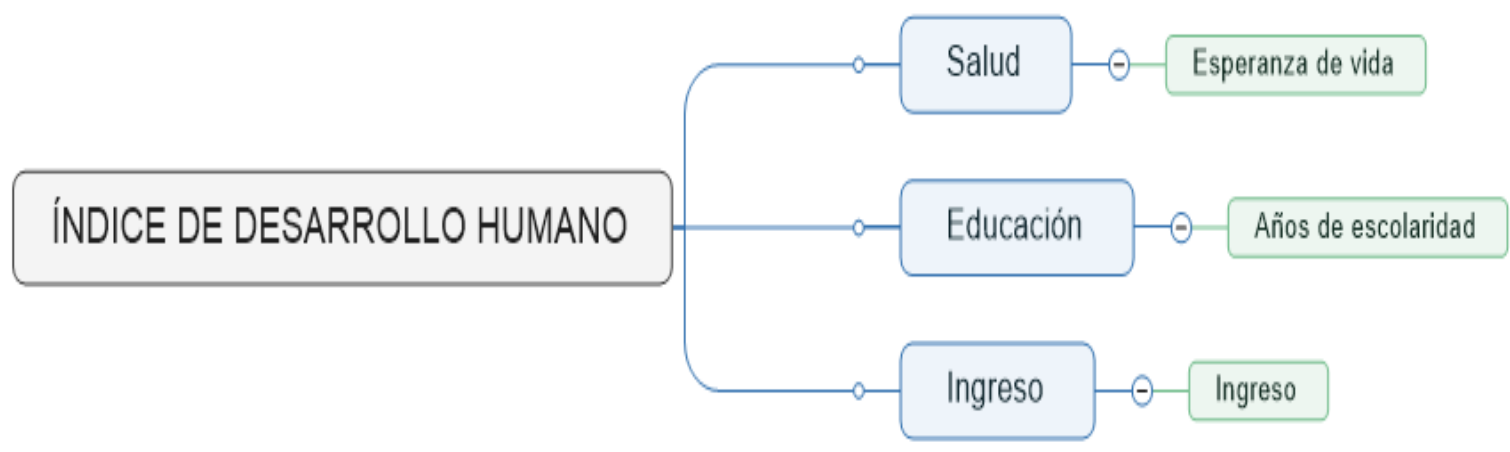

Fuente: Elaboración propia con base al sitio web: https://www.mx.undp.org/content/mexico/es/home/ourwork/ povertyreduction/in_depth/desarrollo-humano.html Nota: De acuerdo al Programa de Naciones Unidas para el Desarrollo (2020), el indicador de ingreso, solamente es incluido como sustituto de las otras variables del desarrollo humano, sin embargo, este no necesariamente refleja una esperanza de vida mayor y saludable.

Como se puede observar en la Figura 1, aún el IDH en apariencia es un tanto corto, por ejemplo, la línea de salud se mide con la simple variable de esperanza de vida en años y deja de lado aspectos como la calidad de vida de las personas, que quizás pueda obtenerse al determinar los años que se vivió completamente saludable, es decir, sin afectaciones físicas y mentales de gravedad, desde luego, entre otras múltiples variables que son mucho más complejas de ser medibles.

En la página web del Programa de las Naciones Unidas para el Desarrollo México (2020), se dice que el parámetro de medición de la línea de la salud, oscila entre los 20 y 83.3 años, respecto al mínimo y máximo de esperanza de vida. Tomando, solamente esa variable el país mexicano se encontraría con un nivel elevado de desarrollo humano, ya que, según el INEGI (2020), se estima que en promedio un mexicano llega a vivir 75.2 años, teniendo variaciones muy leves entre ambos sexos.

Al respecto de lo previamente dicho Cejudo (2006), explica que las capacidades utilizadas en el IDH son las más básicas, sin embargo, las aspiraciones de Amartya Sen van más allá de la elaboración de un índice. "En realidad, su teoría pretende ser un marco conceptual desde el que juzgar no sólo el nivel de vida en un sentido material, sino en general el bienestar entendido como libertad para llevar una vida valiosa." (p. 367)

... en Poder de Paridad de Compra (PPC) expresado en dólares estadounidenses." (Recuperado de: https:// www.mx.undp.org/content/mexico/es/home/ourwork/povertyreduction/in_depth/desarrollo-humano.html) 
DOI: 10.52191/rdojs.2021.207 La seguridad alimentaria, la malnutrición ... Págs.: 8-34

Adelantando un poco se debería preguntar: ¿Cuál es la capacidad real de los mexicanos, para vivir una vida de calidad?

Aspectos determinantes, para la reducción de la calidad de vida en México, tienen ver con la transición nutricional, la inseguridad alimentaria y la malnutrición reflejada en dos condicionantes físicas, por un lado, la desnutrición y por otro, el sobrepeso y la obesidad.

Ante estos fenómenos, que impactan negativamente la salud y el bienestar de millones de mexicanos, las instancias gubernamentales tienen el deber moral y la obligación de actuar a través de políticas públicas, por lo menos, en las propuestas de Sen y Rawls, se trata de expresar algo relacionado a lo dicho, pero esto lo retomaremos más adelante en este manuscrito.

Desde luego, hay quienes como Nozick (2012), sostienen que la intervención del Estado debe ser mínima y que todos somos dueños de nuestras propias decisiones de hacer y de ser, sin embargo, esa libertad de hacer roza en un utilitarismo extremo o radical, el cual, es totalmente opuesto a la propuesta de Amartya Sen. Con esto, concluimos este apartado, en el siguiente trataremos de explicar el por qué consideramos a la inseguridad alimentaria, la malnutrición y la obesidad, como privaciones del pleno desarrollo humano.

\section{La incapacidad de la seguridad alimentaria en México}

Para comenzar esta sección, es preciso dejar claro qué es el enfoque de capacidades propuesto por Sen y Nussbaum. Posteriormente, ofrecer aspectos dirigidos a explicar la inseguridad alimentaria, la malnutrición y la obesidad, que pueden significar signos de incapacidad, y que a su vez, suelen mermar el desarrollo humano de millones de individuos en México y en todo el orbe.

El enfoque de capacidades formulado por Sen, va más allá del modelo de la economía tradicional, el cual, se sustentaba en la libertad de elección de bienes y servicios de consumo, regida por el mercado. También, difiere de la propuesta de Rawls, la cual, se fundamenta en la garantía los bienes primarios o básicos, dejando de lado una infinidad de elementos que contribuyen al desarrollo humano.

El enfoque de la capacidad, seguún Sen (2010), busca las ventajas individuales, desde luego, no en un sentido egoísta, sino desde la perspectiva de ser y poder hacer las cosas que tengan razones para valorarlas sin que estas perjudiquen a terceras personas, a lo anterior se les conoce como libertades sustantivas. En síntesis, se puede decir que se trata de la capacidad de agencia con la que cuentan los seres humanos, para actuar libremente con base a sus valores y sus objeti- 
DOI: 10.52191/rdojs.2021.207 La seguridad alimentaria, la malnutrición ... Págs.: 8-34

vos, empero también a sus oportunidades.

Por su parte Nussbaum (2012), cimienta sus aportaciones teóricas, en relación al enfoque de desarrollo humano o de las capacidades, para esto retoma las obras de Sen, particularmente "Nuevo examen de la desigualdad" y "Desarrollo y libertad". De las que destaca, las capacidades vistas como espacios de ampliación de oportunidades que favorezcan la calidad de vida, dejando a un lado argumentos de la teoría utilitarista. Con lo que, se privilegian aspectos como la salud física, la educación, entre otros.

La autora previamente citada, sostiene que dicho enfoque debe observar o considerar a cada individuo como un fin por sí mismo, y no solamente se centra en el bienestar de la totalidad de los individuos (sociedad), sino también en las oportunidades con las que cuenta cada persona como entidad independiente. Al igual que Amartya Sen, coincide en que el enfoque de las capacidades debe privilegiar la libertad de elección.

Nussbaum (2012) contempla estas oportunidades o libertades sustanciales como un conjunto de bienes aplicables a todas las sociedades. Por lo tanto, afirma que el enfoque de las capacidades tiene como tarea primordial reducir las injusticias y desigualdades sociales.

Para sustentar lo anterior, Nussbaum nos dice que las capacidades no son meras habilidades con las que nacemos o que se adquieren durante los ciclos de nuestras vidas, sino que, son una serie de oportunidades facilitadas por los ámbitos políticos, sociales y económicos. Asimismo, enumera diez capacidades centrales, que deben desarrollarse con la finalidad de que todas las personas tengan como mínimo deseable una vida digna.

La diez capacidades centrales son: vida; salud física; integridad física; sentidos, imaginación y pensamiento; emociones; razón práctica; afiliación; otras especies (animales, plantas, etc.); juego y; control sobre el propio entorno. En la siguiente tabla destacamos dos capacidades que se relacionan directa o indirectamente con la inseguridad alimentaria, la malnutrición y el SPyO.

En la Tabla 1, se muestran dos de las capacidades centrales propuestas por Nussbaum, las cuales, podemos vincularlas con los problemas relacionados a una alimentación insana, en la segunda capacidad es inminente la relación, ya que, se menciona textualmente, sin embargo, en la primera podemos deducir que la reducción de vida y calidad de la misma, pueden convertirse en una incapacidad debido a los efectos negativos de una inadecuada manera de alimentarse. Con esto, daremos paso a lo que hemos nombrado como la incapacidad de una seguridad alimentaria 
DOI: 10.52191/rdojs.2021.207 La seguridad alimentaria, la malnutrición ... Págs.: 8-34

en México, ante esto surge el cuestionamiento:

¿Cómo se interpreta la incapacidad con base a las posturas teóricas de Sen?

En el libro

Tabla 1. Capacidades centrales según Nussbaum
"Desarrollo y libertad",

\section{Definición}

Poder vivir hasta el término de una vida humana de una duración normal; no morir de una forma

Salud física

Poder mantener una buena salud, incluida la salud reproductiva; recibir una alimentación adecuada; disponer de un lugar adecuado para vivir.

Fuente: Elaboración propia con base en Nussbaum (2012, p. 53), donde el texto remarcado en negritas pertenecen a la autora

Amartya Sen plantea el término de privación de la libertad, para referirse a la imposibilidad de las personas de alcanzar un fin, debido a los pocos medios o pocas oportunidades.

En otras palabras, los fines son los funcionamientos, mientras que los medios u oportunidades son las capacidades con las que contamos para ser y hacer. Un ejemplo, quizás un poco simple, sería el consumo de alimento para sobrevivir, en este caso, el alimento es la capacidad y el sobrevivir es el funcionamiento. De manera más acertada y precisa que la nuestra, Nussbaum (2012), ofrece una definición de funcionamiento, el cual "es la realización activa de una o más capacidades." (p. 44)

Para Sen (2000), la falta o la poca libertad es el producto de la violación de derechos humanos fundamentales ${ }^{5}$ y de las incipientes oportunidades a las que tienen acceso un sinnúmero de personas, para lograr mínimas aspiraciones, como lo es la propia sobrevivencia, la alimentación, la convivencia, etc.; sin embargo, cabría agregar que casi todas estas incapacidades pueden ser resueltas o prevenibles.

Por ejemplo, la inseguridad alimentaria es el producto de diversas incapacidades, para com-

5 La alimentación adecuada y la seguridad en la salud, han sido contempladas dentro los derechos humanos universales, en los derechos de los niños, en las garantías sociales enmarcadas en la Constitución Política de los Estados Unidos Mexicanos y en tratados internacionales. 
DOI: 10.52191/rdojs.2021.207 La seguridad alimentaria, la malnutrición ... Págs.: 8-34

prender un poco más al respecto, expondremos la definición de seguridad alimentaria y sus componentes.

La seguridad alimentaria existe cuando todas las personas tienen en todo momento, acceso físico, social y económico a alimentos suficientes, inocuos y nutritivos que satisfacen sus necesidades energéticas diarias y preferencias alimentarias para llevar una vida activa y sana. (Organización de las Naciones Unidas para la Alimentación y la Agricultura, 2011, p. 1)

De acuerdo al Consejo Nacional de Evaluación de la Política de Desarrollo Social (CONEVAL, 2010), las dimensiones de la seguridad alimentaria son:

- Disponibilidad de alimentos: se dice que es la primera fase de la seguridad alimentaria, la cual, consiste en la creación de alimentos sin procesar y procesados. Este principio, apela la existencia y disposición de alimentos suficientes por lo menos durante todo un año.

- Consumo de alimentos: hace referencia a los alimentos ingeridos en cada familia, sin importar su origen (autoproducción, intercambio, apoyos, adquisición, etc.).

- Aprovechamiento biológico de los alimentos: este tiene una dependencia directa con la condición de salud de cada persona.

- Estado nutricional de cada persona: en esta dimensión no solo se contempla al estado nutricional de los individuos, sino que, incorpora elementos como servicios de salud, ambientes e higiene.

En la siguiente figura, se muestran los componentes esenciales de la seguridad alimentaria de acuerdo a la Organización de las Naciones Unidas para la Alimentación y la Agricultura (FAO, 2011), los cuales, difieren un poco a los presentados por el CONEVAL. 
Año 16, Vol. 11, número 21, enero-junio 2021

DOXA
Sección: Ciencias Sociales

DOI: 10.52191/rdojs.2021.207 La seguridad alimentaria, la malnutrición ... Págs.: 8-34

Figura 2. Componentes de la seguridad alimentaria

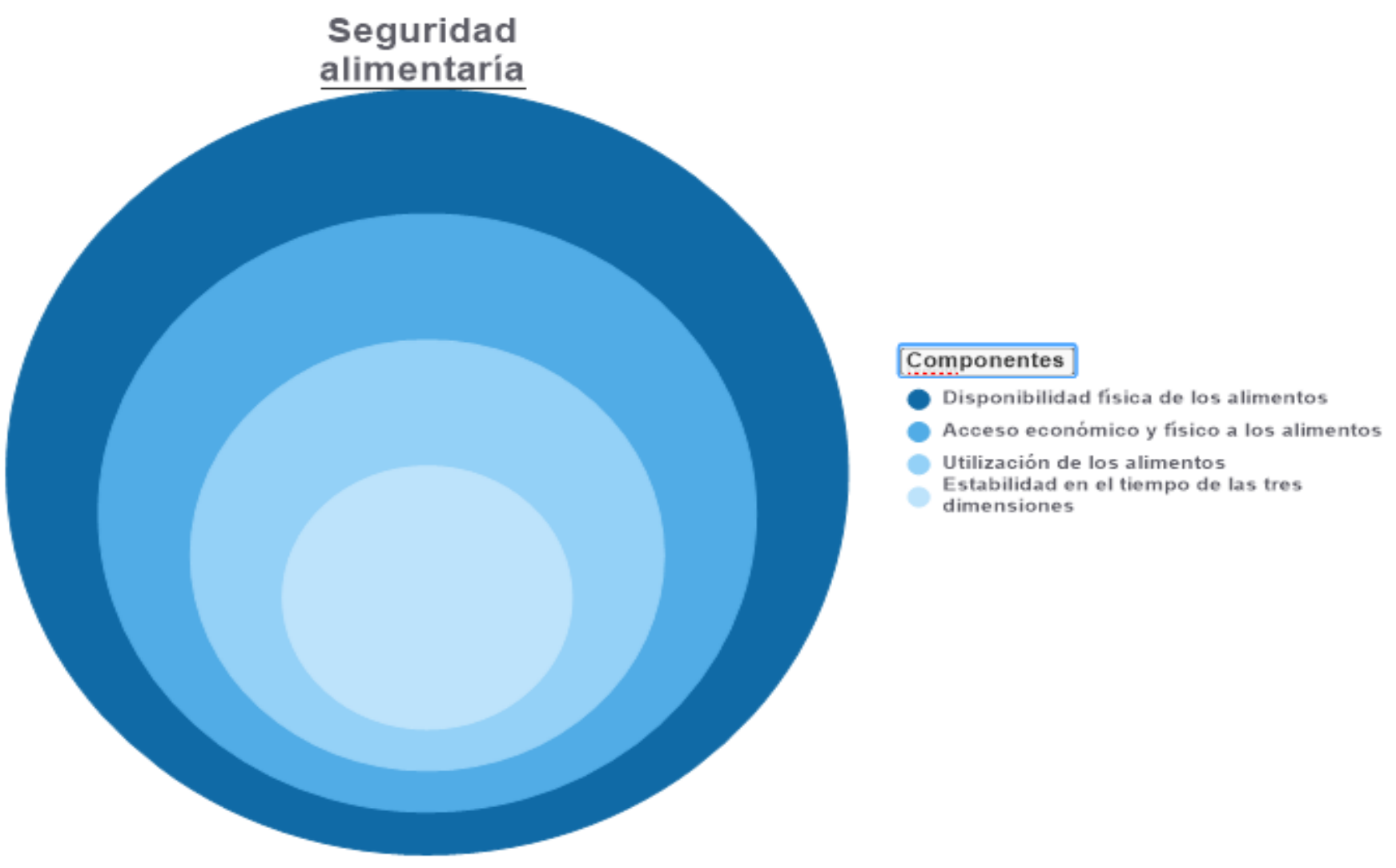

Fuente: Elaboración propia con base a: Guía Práctica "La Seguridad Alimentaria: información para la toma de decisiones".

En la Figura 2, se encuentran una serie de potenciales incapacidades o falta de capacidades, en relación a la disponibilidad física de los alimentos, la mayor preocupación no es por la gran variedad de productos alimentarios que se comercializan, sino, por la calidad de los mismos, los cuales se ofertan en los grandes supermercados, en los mercados locales, en las tiendas de conveniencia e incluso en las tienditas ubicadas en nuestras colonias.

Si bien es cierto, se respeta el elemento de la libre elección o la capacidad de decidir qué comer, el verdadero problema radica en el tipo de alimentos que se ponen a la venta. La Organización Panamericana de la Salud (OPS 2015), hace mención a un fenómeno llamado transición nutricional, que consiste en el cambio de patrones alimenticios y físicos, derivados en gran medida por la globalización, las políticas de libre mercado y la urbanización. En este contexto, el organismo internacional referido, hace hincapié en la sustitución de alimentos caseros con altos contenidos nu- 
DOI: 10.52191/rdojs.2021.207 La seguridad alimentaria, la malnutrición ... Págs.: 8-34

tricionales por los llamados productos ultraprocesados ${ }^{6}$, los cuales, regularmente son instantáneos, tienen un buen sabor y sobre todo se localizan con facilidad.

La mayoría de los productos que se venden en los supermercados, especialmente en los pasillos centrales y en las cabeceras de los pasillos, son ultraprocesados, lo mismo que la mayoría de los productos de las "tiendas de conveniencia" y los locales de comida rápida. (Organización Panamericana de la Salud, 2015, p. 5)

De acuerdo a la Gaceta UNAM (2019), México para el año citado, ocupaba el primer lugar en consumo de refrescos en todo el mundo, se dice que, en promedio cada familia gasta el $10 \%$ de sus ingresos totales en bebidas de ese tipo, asimismo, se afirma que anualmente se consumen alrededor 163 litros por persona, superando incluso a países como Estados Unidos, quien ocupa el segundo lugar con 118 litros. Otro dato aún más alarmante, lo dio a conocer en López-Gatell, en la Conferencia de Prensa del día 17 de julio 2020, en la cual, dio a conocer que en el estado de Chiapas, la media estadística en la ingesta de refresco por habitante es de 821.25 litros.

En este mismo sentido, la Organización de las Naciones Unidas para la Alimentación y la Agricultura (FAO, 2019), informó que el país mexicano es uno de los mayores consumidores de productos alimenticios ultraprocesados ${ }^{7}$, ya que, se calcula que cada mexicano en promedio consume 581 gramos de alimentos industrializados al día.

Sin lugar a dudas, lo anterior se puede considerar como una incapacidad o falta de oportunidades, en referencia a la disponibilidad de alimentos sanos y nutritivos, en comparación a la oferta de productos industrializados. Aunado a esto, la mercadotecnia y la publicidad en diversos medios de comunicación, propician un consumo desmedido de "comida chatarra". Con estos datos, se puede deducir que el funcionamiento queda restringido a la saciedad, pero no a la nutrición adecuada.

Algo que se destaca, es que la población infantil es más susceptible a la persuasión en el consumo de productos industrializados, asimismo, los menores son expuestos a ambientes obesogéni$\cos { }^{7}$ en los cuales, con facilidad encuentran dichas mercancías. Al respecto, la organización no

6 Los productos ultraprocesados son formulaciones industriales elaboradas a partir de sustancias derivadas de los alimentos o sintetizadas de otras fuentes orgánicas. (Organización Panamericana de la Salud, 2015, p. 5).

7 Entre los que podemos encontrar golosinas, frituras, papas fritas, snacks, barraras energéticas, chocolates, etc.

8 Entorno que fomenta la ingesta calórica elevada y el sedentarismo. Se tienen en cuenta los alimentos disponibles, asequibles, accesibles y promocionados; las oportunidades para practicar una actividad física; y las normas sociales en relación con la alimentación y la actividad física. (OMS, 2016, p. v) 
DOI: 10.52191/rdojs.2021.207 La seguridad alimentaria, la malnutrición ... Págs.: 8-34

gubernamental "Alianza por la Salud Alimentaria (2018), nos dice que "las niñas y los niños son el grupo social más vulnerable a los efectos de la publicidad y en muchos casos pueden convertirse en dependientes del mercado del consumo." (p. 6)

Un claro ejemplo de la alta ingesta de alimentos ultraprocesados o industrializados, por parte de la población infantil, fue el estudio realizado por el Instituto de Salud Pública (INSP), efectuado en el año 2018, en el cual se daba a conocer que los niños mexicanos en edades de dos a nueve años, consumían en promedio un $38.6 \%$ del total de calorías ingeridas durante el día, provenientes de dichos productos alimenticios procesados o ultraprocesados, tales como golosinas, snacks, papas fritas, jugos, refrescos, entre otros.

Lo anterior, en gran medida obedece al alto bombardeo publicitario al que están expuestos los menores, ante lo mencionado el propio Instituto Nacional de Salud Pública, señala en uno de sus estudios efectuado entre los años 2012 a 2013, se registraron un total de 12311 anuncios de los cuales un $23.3 \%$ correspondían a alimentos y bebidas industrializados. Además:

Se encontró que el horario con mayor número de comerciales fue entre las 16:00 y 19:00 hrs. Los productos más promocionados durante este horario fueron las botanas dulces (34.4\%) y las bebidas azucaradas $(21.3 \%)$. Se encontró que los anuncios de botanas dulces fueron dirigidos principalmente a la población infantil;

De acuerdo al tipo de programa, se observó que en las caricaturas se presentó un mayor porcentaje de anuncios de cereales azucarados (16.6\%) y de botanas dulces (53.5\%). (INSP, 2021, recuperado de: https://www.insp.mx/epppo/blog/2984-publicidad-alimentos-bebidas.html)

También, es pertinente dejar en claro que este fenómeno de transición nutricia, no es exclusivo de algún grupo de edad o sector de la población, sin embargo, diversas organizaciones internacionales como la OMS, la OPS y la UNICEF, encuentran una correlación entre el ingreso y el tipo de consumo. Lo anterior, está vinculado al componente de acceso económico y físico a los alimentos, el cual, puede ser consecuencia de las desigualdades o falta de oportunidades para acceder a comida saludable y nutritiva. Aquí, la privación económica y la pobreza en ingreso de gran número de personas son factores determinantes.

Según el Consejo Nacional de Evaluación de la Política de Desarrollo Social (2020), en México para el año 2018 , se estimaba que el $48.8 \%$ de la población o lo equivalente a decir 61.1 millones de personas, percibían un ingreso inferior a la línea de pobreza por ingresos, es decir, por debajo del valor económico de la canasta básica, de los bienes y de los servicios. Mientas que, un 
DOI: 10.52191/rdojs.2021.207 La seguridad alimentaria, la malnutrición ... Págs.: 8-34

16.8\% (21 millones de personas), tenían un ingreso inferior a la línea de pobreza extrema, lo que significa, que no poseían la capacidad de por lo menos, solventar la canasta alimentaria básica ${ }^{9}$. Y finalmente, el 20.4\% (25.5 millones de personas), no contaba con acceso a una alimentación adecuada, ya que, ni utilizando todo el ingreso disponible podían adquirir la totalidad de los alimentos básicos.

De acuerdo a un boletín emitido por la Secretaría del Trabajo y Previsión Social (2019), se afirma que para el año 2020 , el salario mínimo ${ }^{10}$ pasó a 123.22 pesos en casi todas las entidades federativas, a excepción de aquellas ubicadas en la Zona Libre de la Frontera, en donde, se percibía un sueldo mínimo de 185.56 pesos al día.

Mientras, que la canasta alimentaria de acuerdo a CONEVAL (2020), cuesta o tiene un precio de alrededor de $\$ 1,640$ al mes, empero, según Herrera (2020), tan solo contempla los siguientes productos y cantidades: 10 litros de leche (\$183.9), 10 barras de pan de $500 \mathrm{gr}$. (\$293.10), arroz 1 $1 / 2 \mathrm{~kg}$. (28.68), 20 huevos (\$46.47), queso $1 \mathrm{~kg}$. (\$98.88), pollo y carne de res $6 \mathrm{~kg}$. (\$672.18), frutas $6 \mathrm{~kg}$., y verduras $8 \mathrm{~kg}$. (\$171.74). Con estos datos, observamos que cerca del $50 \%$ del ingreso total de los trabajadores con salario mínimo se destina a alimentos limitados en elección y en cantidad.

La privación o falta de oportunidades, para un mayor ingreso son causas directas de la incapacidad de acceso a alimentos nutritivos, por lo que, muchas veces estos son sustituidos por productos alimenticios ultraprocesados, que suelen ser más económicos. En referencia a esto, la Organización de las Naciones Unidas para la Alimentación y la Agricultura (2020), acepta que los alimentos orgánicos tienen costos mayores, debido a varios motivos, entre los que se destacan:

- Se tiene una oferta limitada con relación a la demanda.

- Los costos de producción son elevados en comparación a los productos industrializados, los cuales, son producidos a grandes escalas.

- $\quad$ Existe una gran especulación respecto a los precios.

- La comercialización y distribución suele ser limitada.

Con respecto, al componente de la utilización de los alimentos, se sugiere que cada persona debe ingerir porciones adecuadas y ricas en nutrimentos de acuerdo a la edad, la talla, la actividad

9 De acuerdo a la Secretaría de Agricultura y Desarrollo Rural (2020), la canasta básica de alimentos consta de 40 artículos. Revisar en: https://www.gob.mx/canastabasica

10 Desde luego, se habla del salario percibido en trabajos formales. 
DOI: 10.52191/rdojs.2021.207 La seguridad alimentaria, la malnutrición ... Págs.: 8-34

física, entre los aspectos a destacar. Lo que, se puede decir es que en México debido a las carencias socioeconómicas en las que se encuentra la mayor parte de la población, la alimentación no es balanceada en la mayoría de los casos y, si a eso, añadimos el consumo desmedido de productos ultraprocesados, indiscutiblemente hablamos de una incapacidad de la utilidad física y nutrimental de nuestro cuerpo, con respecto a los alimentos ingeridos en el transcurso del día.

Finalmente, el componente de la estabilidad en el tiempo de las tres dimensiones de la seguridad alimentaria, se refiere a la conjunción permanente de los tres componentes previamente referidos. Si todos o alguno de ellos falla, necesariamente hablamos de inseguridad alimentaria, y esta a su vez, genera un nuevo problema conocido como malnutrición, que sería la incapacidad de alimentarse sana y nutritivamente.

La malnutrición resulta de deficiencias, excesos o desequilibrios en el consumo de macro o micronutrientes. La malnutrición pueda ser un resultado de la inseguridad alimentaria, o puede estar relacionada con factores no alimentarios, como prácticas inadecuadas de cuidado de los niños, servicios de salud insuficientes o un medio ambiente insalubre. (FAO, 2011, p. 3)

La malnutrición como se ha mencionado anteriormente, deriva en dos problemas de salud, el primero es la insuficiencia ponderal (desnutrición) ${ }^{11}$, mientras que el segundo es el sobrepeso y la obesidad. Nosotros para este artículo, tan solo nos basaremos en lo concerniente al SPyO. Debemos aclarar que tanto la obesidad como el sobrepeso, son el resultado de la acumulación elevada de grasa, pero en niveles diferentes.

De acuerdo a la Encuesta de Salud y Nutrición (ENSANUT, 2018), el 37.9\% de las niñas y los niños presentaban sobrepeso u obesidad. Mientras que, el grupo de edad de 12 a 19 años, presentaron una prevalencia combinada de SPyO de $35.8 \%$, para ambos sexos. En tanto, la población de 20 o más años, registró que un $75.2 \%$ de hombres y mujeres tenían obesidad o sobrepeso.

Otro dato, a resaltar es que en todos los rangos por edad, las prevalencias de SPyO fueron mayores en las áreas urbanas ${ }^{12}$ en comparación a las zonas rurales del país mexicano. Con un $37.9 \%$ y un $29.7 \%$, respectivamente.

La vida en las ciudades y las crecientes presiones derivadas de la comercialización masiva, la disponibilidad de productos comestibles malsanos y el acceso a la automatización y al transporte influyen sobre el modo de vida y afectan directamente a la salud. (Organización Mundial de la Salud, 2020)

11 De acuerdo a la FAO (2019), por cada persona hambrienta, existen seis personas con obesidad o sobrepeso. 12 Según la OMS, actualmente (2020) más de la mitad de la población a nivel mundial reside en ciudades y se espera que, para el año 2050 , llegue a un $70 \%$. 
DOI: 10.52191/rdojs.2021.207 La seguridad alimentaria, la malnutrición ... Págs.: 8-34

Asimismo, no ésta por demás recordar que México ocupa el primer lugar de obesidad infantil y el segundo puesto de adultos obesos a nivel mundial.

Quizás, ustedes se cuestionen: ¿esos datos qué relevancia tienen con respecto a la teoría de las capacidades de Amartya Sen?

Por increíble que parezca, la respuesta viene desde las ciencias de la salud, para Salazar, Salazar y Pérez (2015), existen dos tipos de obesos los funcionales y los disfuncionales, los primeros son aquellos que solo presentan unos kilos demás (sobrepeso) y que tienen la capacidad de realizar funciones básicas y laborales por sí mismos, aunque con ciertas limitantes; en tanto los segundos, son incapaces de realizar tareas rutinarias sin ayuda de otra u otras personas.

Desde luego, quienes son obesos funcionales, también corren el riesgo de desarrollar en un futuro algún tipo de enfermedad crónico-degenerativa no transmisible como diabetes, hipertensión, distintos tipos de cáncer, entre otras. Cuando alguna persona se enferma, su incapacidad incrementa sustancialmente, puede sufrir, por ejemplo, despido del trabajo por su baja productividad, descenso en su ingreso per cápita, la disminución de actividades rutinarias, rechazo social, pero lo más delicado es la disminución de la esperanza de vida y de la calidad de vida.

Un ejemplo, referente a lo previamente dicho es la alta comorbilidad de personas contagiadas por Covid-19 y que presentan algún tipo de enfermedad crónico-degenerativa, de hecho siendo más precisos la obesidad es considerada una enfermedad por si sola. Para dejar más claro lo que queremos decir, presentamos una gráfica con las cifras oficiales de las defunciones por coronavirus registradas en México.

En la Gráfica 1, se observan las cuatro principales comorbilidades, relacionadas a las muertes registradas por Covid-19, tres de ellas tienen una relación directa con el exceso de peso, las cuales son obesidad, diabetes e hipertensión. Esta pandemia, vino a recordarnos lo enfermos que estamos los mexicanos, desde hace ya muchos años.

Sin lugar a dudas, el sobrepeso y la obesidad han sido determinantes para el deterioro acelerado y constante de las capacidades. Por lo tanto, es un problema que se tiene que resolver desde la raíz. La seguridad alimentaria en un sentido un tanto romántico, tendría que ser considerada como un bien público, en el que, no se excluya y se rivalice a ninguna persona. Es cierto, que la alimentación es un derecho humano universal, lastimosamente millones de personas en México y en el mundo son incapaces de acceder a los alimentos inocuos y nutritivos. 


\section{Gráfica 1. Principales comorbilidades}

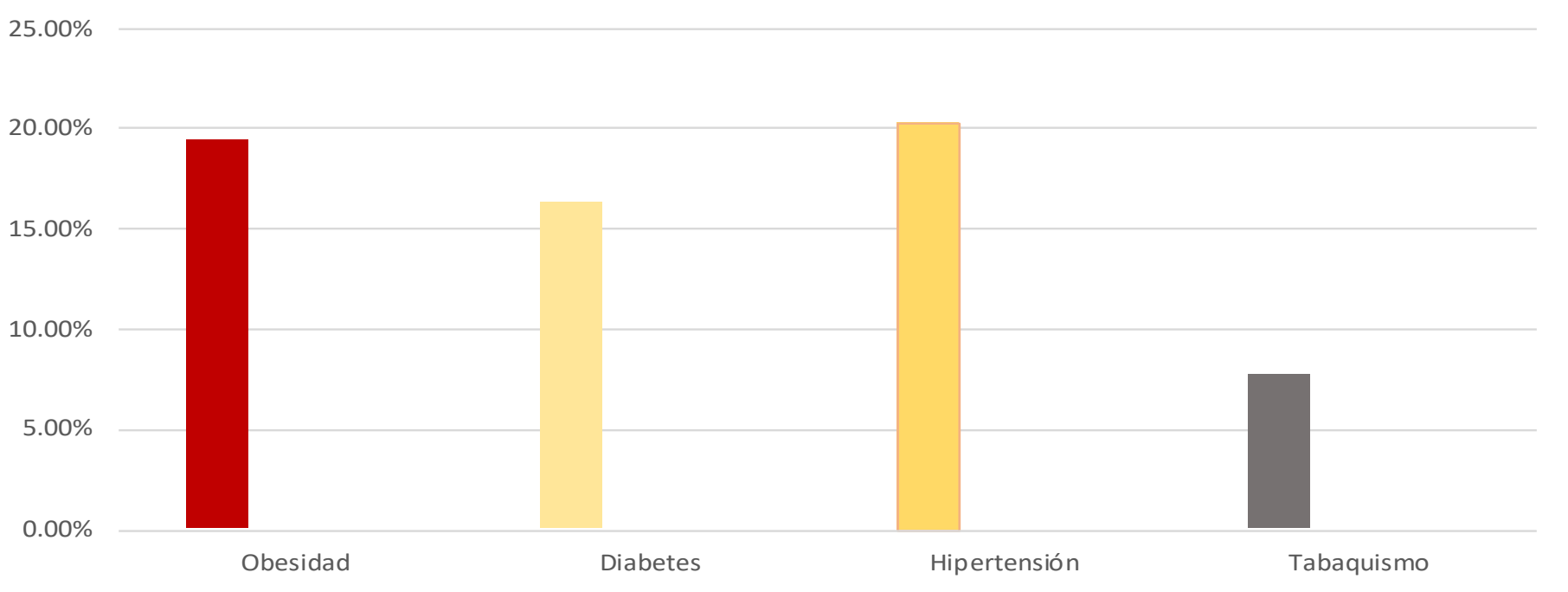

Fuente: Elaboración propia con base al sitio web: https://coronavirus.gob.mx/datos/

\section{La dualidad de la justicia y la seguridad alimentaria}

En este apartado, se realiza una exposición muy breve de los elementos fundamentales de la idea de justicia de dos de los grandes exponentes contemporáneos del tema, como lo son Amartya Sen y John Rawls. Esto es importante, ya que, se observará cuál de las propuestas podría ser más benéfica en el impacto de las políticas públicas y sociales, en este caso dirigidas a garantizar la seguridad alimentaria y, por ende, la disminución de los altos índices de malnutrición, reflejados en las prevalencias de sobrepeso y obesidad en México.

Comenzaremos con la idea de justicia propuesta por Rawls (2002), quien se reconoce por ser

un contractualista moderno. Él, supone que los individuos realizan un contrato, en el que, determinan lo moralmente deseable para la totalidad de la sociedad. Asimismo, cree en el funcionamiento armónico de las instituciones sociales y políticas; las cuales, tienen la labor de disminuir las desigualdades entre individuos.

El autor previamente referido, establece que existen dos elementos esenciales de la justicia los cuales son la libertad y la equidad. En relación al segundo elemento, como lo dijimos previamente, Rawls (2002), es consciente de las desigualdades existentes entre las personas. Por lo tan- 
DOI: 10.52191/rdojs.2021.207 La seguridad alimentaria, la malnutrición ... Págs.: 8-34

to, centra su atención en los individuos con mayores desventajas y para determinar dichas carencias, tan solo se concentra en el acceso a los bienes primarios, dejando de lado otros aspectos como el bienestar y el desarrollo, a los que da por sentado.

El propio Amartya Sen (2010), reconoce que muchas de estas desventajas o incapacidades pueden ser prevenidas con la intervención de las instancias gubernamentales correspondientes. La diferencia principal entre Rawls y Sen, radica en el que el primero da una gran importancia a lo establecido formalmente, mientras el segundo, añade la importancia de las libertades sustanciales.

En relación a la propuesta de justicia de Sen (2010), es más ambiciosa y no se limita a los bienes básicos como en el caso de Rawls, lo que, él propone es la generación de capacidades de cada uno de los individuos para hacer y ser. Pero, esto no significa que no sugiera disminuir a través de estímulos los problemas que privan de las libertades a los seres humanos como son la pobreza, el inacceso a la salud, la inseguridad alimentaria, entre otros tantos. En palabras de Antón (2013), se puede decir que:

La idea de justicia como equidad en Rawls está basada en la 'imparcialidad' de la correspondencia respecto de los méritos o proporcionalidad. La posición original con el 'velo de la ignorancia' es partir de cero (sin contar intereses y opiniones) y es cuando surgen los principios de la justicia. Después viene el 'trato igual', apoyando a los más desfavorecidos con una base de bienes primarios mínimos. En Sen, ese concepto de equidad es 'objetividad' o neutralidad junto con tener en cuenta a los 'otros' y sus particularidades. Es decir, contempla sus condiciones concretas de existencia, sus necesidades, para estimular las capacidades que le puedan hacer libres y tomar sus decisiones. (p.192)

En cuanto a los en los programas sociales en el país mexicano, Florido (2020) indica que, a partir de la implementación del Programa de Educación, Salud y Alimentación (PROGRESA) ${ }^{13}$ en 1997, la mayor parte de las políticas sociales en México dieron un nuevo viraje hacía la teoría de las capacidades, aunque pudieran ser una combinación de los enfoques de Rawls y Sen, pero más inclinadas al último teórico mencionado. Según el CONEVAL (2019), se busca eliminar algún tipo de carencia social, a través estímulos económicos y alimentarios principalmente, esto con la intención de fomentar el desarrollo humano y las capacidades de los beneficiarios de todas las edades.

Este abordaje se basó en el argumento de que era necesario focalizar las acciones de política social hacia la población con mayores vulnerabilidades sociales (Grosh, 1994) y, con base en el enfoque de

13 De acuerdo a la Coordinación Nacional de Becas para el Bienestar Benito Juárez (2020), para el año 2002 el programa social tuvo algunas modificaciones incluido el nombre, el cual, cambio a OPORTUNIDADES. Para el 2014, se volvió a renombrar como Programa de Inclusión Social PROSPERA. Y finalmente, con la administración de López Obrador, se le ha llamado Becas para el Bienestar Benito Juárez. 
DOI: 10.52191/rdojs.2021.207 La seguridad alimentaria, la malnutrición ... Págs.: 8-34

capacidades propuesto por Sen (1983) y Levy (1991), destacó la importancia de asegurar condiciones adecuadas de salud, nutrición y educación entre la población más pobre, con el objetivo de que esté en condiciones de desarrollar sus capacidades. (CONEVAL, 2019, p. 423)

Algunas consecuencias no buscadas o imprevistas, como las llamaría Merton (1992), que se observan en este tipo de políticas es que se centran más en la cobertura, que en el impacto real que pueda llegar a tener en el beneficiario. Por ejemplo, tras años de analizar a programas escolares en los que se ofrece el servicio de alimentación, encaminados a la reducción de los altos índices de malnutrición, nos dimos cuenta de múltiples deficiencias e irregularidades, que van desde el robo de alimentos, hasta la falta de registros acerca de la condición física de los estudiantes. Al respecto de esto, Martínez (2019), identifica cinco fallas que han caracterizado a los programas sociales en México, las cuales son:

La insípida coordinación y cooperación entre las dependencias gubernamentales de los tres órdenes de gobierno (federal, estatal y municipal), lo anterior deriva en la constante duplicidad de programas, y por ende, el inminente despilfarro de los recursos públicos.

La opacidad en la transparencia y rendición de cuentas, lo que termina provocando corrupción, así como, la utilización de los programas con fines electorales, creando un clientelismo asiduo.

La poca consecución de los objetivos y metas de los programas sociales.

Los problemas con las asignaciones de presupuesto, dirigidas a los programas sociales y los bajos resultados en el desempeño.

La poca relación de los programas sociales con la universalización de protección social, la garantía de los derechos sociales y las bases económicas que generan inevitablemente los distintos niveles de pobreza, es decir, la dualidad entre los ingresos y el consumo.

En el caso de los programas con comedores escolares, lo que se busca idealmente, es que los infantes desarrollen las capacidades a través de la educación y la generación de hábitos alimenticios saludables, asimismo, se pretende que trasciendan de los entornos educativos a los hogares. Por supuesto, pensando en un futuro, pero también enfocados en la inmediatez del presente que vivimos. Podemos decir que, un niño con capacidades es un niño con múltiples oportunidades.

El Servicio de Alimentación debe contribuir a que las alumnas y alumnos tomen decisiones informadas sobre su alimentación e higiene personal y familiar, adopten un enfoque preventivo que les posibilite identificar las ventajas de cuidar su cuerpo, tener una alimentación correcta y practicar actividades físicas con regularidad. (SEP, 2017, p. 29) 
DOI: 10.52191/rdojs.2021.207 La seguridad alimentaria, la malnutrición ... Págs.: 8-34

Un aspecto más, en relación a la teoría de la justicia de Sen, que no podemos dejar de lado es lo concerniente a los funcionamientos, quisiéremos tratar explicarlo con un ejemplo sencillo y de esta manera subrayar que no todos tenemos los mismos objetivos o valoraciones sobre lo que deseamos. A continuación, mostramos un diagrama en el que se detalla una sola capacidad y con distintos funcionamientos.

Figura 3. Funcionamientos de la capacidad de una alimentación sana

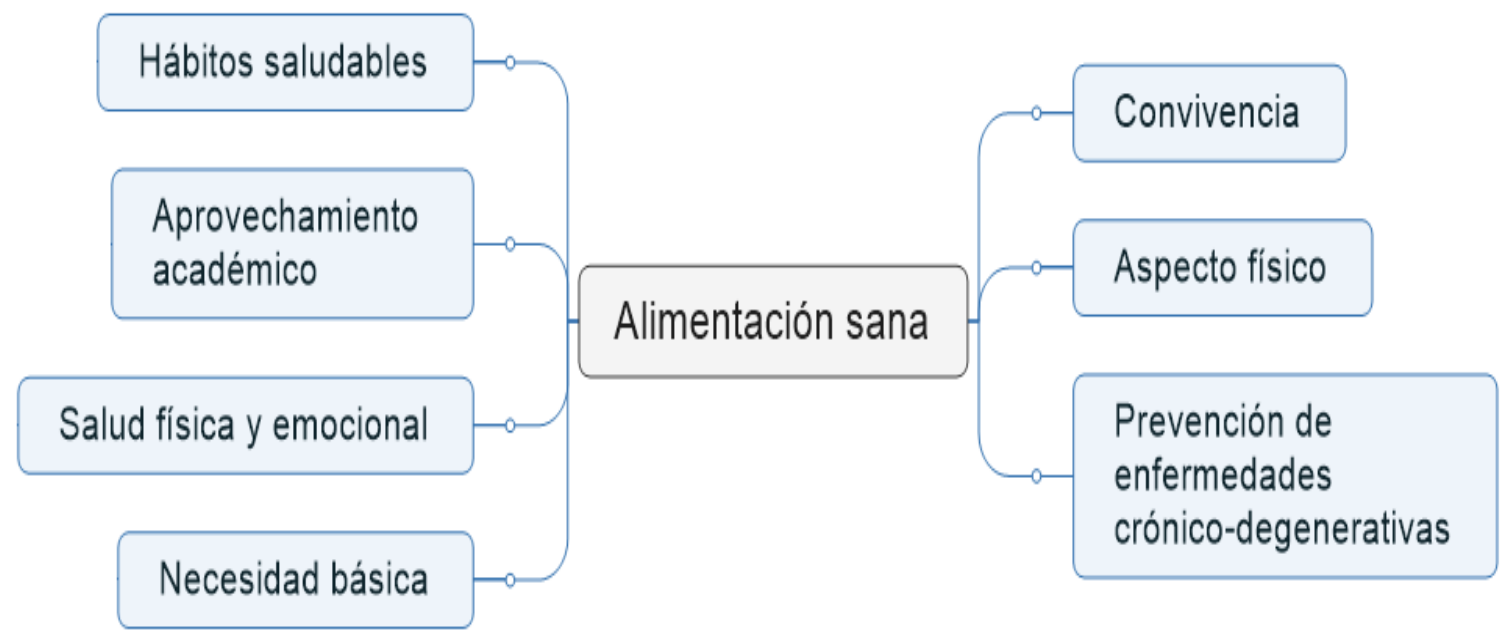

Fuente: Creación Propia.

En la Figura 3, se expone un ejemplo de una capacidad, que en este caso es alimentarse sanamente, además incluimos algunos funcionamientos posibles, por ejemplo, quizás alguien se alimenta sano para prevenir las enfermedades crónico-degenerativas, otro tal vez, elige este modo de alimentarse para mantener un aspecto físico deseable, etc. Para Sen (en Cejudo, 2006), "el funcionamiento es algo que se logra, mientras que la capacidad es la facultad de lograr." (p. 368)

Al interpretar la Figura 3, bajo el enfoque de justicia de Rawls, posiblemente se limitaría a definir a la alimentación como el medio, para satisfacer una necesidad vital, sin ir más allá. Posiblemente, esto es lo que ocurre realmente en los programas con comedores escolares, sustentamos lo dicho, en nuestras experiencias personales, sujetas a la objetividad y a la ética profesional. En una ocasión, nos entrevistamos con una servidora pública de la Secretaría de Educación, a la cual, le preguntamos: ¿cuáles son los alimentos ofrecidos a los niños por las mañanas?, nos contestó diciendo galletas y leche. Lo que queremos decir, es que se alimenta a los menores con la intención de saciar el hambre, pero descuidando aspectos como el aporte en la calidad nutricional, así 
DOI: 10.52191/rdojs.2021.207 La seguridad alimentaria, la malnutrición ... Págs.: 8-34

como, concientizar y generar hábitos saludables permanentes en los infantes.

\section{Conclusiones}

Podemos concluir, que la inseguridad alimentaria, la malnutrición y la obesidad, son el reflejo del incipiente desarrollo humano en México. Y que estas incapacidades, han repercutido en la salud física y emocional, en los ingresos, en los elevados gastos para sortear los tratamientos, en la exclusión social, empero, principalmente han reducido la esperanza y calidad de vida de millones de mexicanos y mexicanas.

Algo positivo, es que la mayor parte de las incapacidades señaladas en este escrito, son prevenibles, sobre todo si son atendidas desde edades tempranas, es decir, desde la infancia. Se debe tener como objetivo principal, la formación de capacidades en los niños y las niñas, para que en su futuro tengan un desarrollo humano mejor que el nuestro.

Si bien es cierto, que la obesidad y el sobrepeso han sido identificados como un problema de salud pública mundial. De acuerdo a la Organización Panamericana de la Salud (OPS, 2020), no se puede ofrecer una solución universal, debido a que las características entre países o regiones son heterogéneas. Por lo que, resulta primordial identificar las necesidades primarias e inequidades en el ámbito de la salud y alimentación. Para lo anterior, se sugiere el constante monitoreo de los patrones alimenticios y sus cambios, ya que, de esta forma se pueden formular supuestos o hipótesis, asimismo, evaluar el impacto de los programas y políticas en materia de sanidad y alimentación.

Lo que es un hecho, es la urgencia de desarrollar políticas públicas integrales dirigidas a la disminución de las altas prevalencias de sobrepeso y obesidad. Tenemos en claro, que un programa como el de comedores escolares, quizás pueda tener poco impacto, sin embargo, si este se acompaña de restricciones en la venta de alimentos con altos contenidos calóricos al interior de las escuelas y en las periferias, si aplican políticas fiscales dirigidas a las grandes compañías o corporaciones de alimentos industrializados, si se generan los apoyos necesarios al sector primario, entre otros tipos de medidas que se entrelacen unas con otras, hasta entonces podremos hablar de una política integral en materia de seguridad alimentaria.

Un gran paso se ha dado, con la aprobación del nuevo etiquetado frontal, en el cual, se informa de manera clara a los consumidores acerca del contenido de los productos industrializados empaquetados y embotellados, quizás esa capacidad de estar informado pueda repercutir positivamente en la alimentación de los mexicanos. 
DOI: 10.52191/rdojs.2021.207 La seguridad alimentaria, la malnutrición ... Págs.: 8-34

Finalmente, quisiéremos expresar que nosotros coincidimos con Nussbaum, la cual, expresa que "mejorar la calidad de vida de las personas exige decisiones políticas inteligentes y la participación dedicada de muchos individuos." (2012, p. 15).

En este sentido, nos atrevemos a decir que la seguridad alimentaria no solo se trata de un asunto de derechos humanos universales, los cuales, son plasmados en tratados y pactos internacionales, así como, en distintas constituciones de diversos países, tal es el caso de México, que desde la Constitución Política de los Estados Unidos Mexicanos de 1917, se establecieron por escrito las garantías sociales, entre las cuales, se estipula en su artículo cuarto el derecho de todos los mexicanos a una alimentación de calidad nutritiva y en cantidades suficientes. Podemos concluir, que más allá de lo escrito en un papel, que en diversas ocasiones no se lleva a la práctica, eliminar la inseguridad alimentaria de millones de personas en México y el mundo, se trata de un tema de justicia y moral.

\section{Referencias}

Antón, A. (2013). Igualdad y Libertad: Fundamentos de la Justicia Social. Revista internacional de educación para la justicia social, 2(1), 173-194.

Asociación de Academias de la Lengua Española. (2020). Real Academia Española. Obtenido de https://www.rae.es/

Basu, K. (2002). Sobre las metas del desarrollo. En G. M. Stiglitz, Fronteras de la Economía del Desarrollo. El Futuro en perspectiva (págs. 49-74). Washington, D.C.: Banco Mundial.

Salazar, B., Salazar, M., Tamayo, R. (2015). ¿Gorditos o enfermos? La obesidad en niños y adolescentes. México: Fondo de Cultura Económica, El Colegio Nacional e Instituto Nacional de Salud Pública.

Cámara de Diputados del H. Congreso de la Unión. (2021). Constitución Política de los Estados Unidos Mexicanos. México: Secretaría de Servicios Parlamentarios.

CÁMARA DE DIPUTADOS DEL H. CONGRESO DE LA UNIÓN. (2019). CONSTITUCIÓN POLÍTICA DE LOS ESTADOS UNIDOS MEXICANOS. México: CÁMARA DE DIPUTADOS DEL H. CONGRESO DE LA UNIÓN.

Cejudo, R. (2006). Desarrollo humano y capacidades. Aplicaciones de la teoría de las capacidades de Amartya Sen a la educación. Revista española de pedagogía, 64(234), 365-380. 
DOI: 10.52191/rdojs.2021.207 La seguridad alimentaria, la malnutrición ... Págs.: 8-34

Consejo Nacional de Evaluación de la Política de Desarrollo Social. (2010). Dimensiones de la seguridad alimentaria: Evaluación Estratégica de Nutrición y Abasto. México: CONEVAL,.

Consejo Nacional de Evaluación de la Política de Desarrollo Social. (2020). CONEVAL. Obtenido de Medición de la pobreza: https://www.coneval.org.mx/Medicion/Paginas/Glosario.aspx

Coordinación Nacional de Becas para el Bienestar Benito Juárez. (2020). Gobierno de México. Obtenido de ¿Qué es PROSPERA Programa de Inclusión Social?: https://www.gob.mx/ becasbenitojuarez/documentos/que-es-prospera\#: :text=PROSPERA\%20es\%20un\% 20programa\%20de,las\%20personas $\% 20$ beneficiarias $\% 20$ con $\% 20$ proyectos

Delgado, S. (28 de Octubre de 2019). Gaceta UNAM. Obtenido de México, primer consumidor de refrescos en el mundo: https://www.gaceta.unam.mx/mexico-primer-consumidor/

Herrera, E. (18 de Mayo de 2020). LJA.MX. Obtenido de La canasta básica mexicana y su valor/el apunte: https://www.lja.mx/2020/05/la-canasta-basica-mexicana-y-su-valor-el-apunte/

Florido, Á. (05 de Julio de 2020). Programas sociales en México. (L. Arias, Entrevistador)

Hernández, G., de la Garza, T., Zamudio, J., Yaschine, I. (Coord.). (2019). El ProgresaOportunidades-Prospera, a veinte años de su creación. México: Consejo Nacional de Evaluación de la Política Social.

Grupo Copesa. (2020). La Tercera. Obtenido de Amartya Sen: "El desarrollo es más que un número": https://www.latercera.com/noticia/amartya-sen-el-desarrollo-es-mas-que-un-numero-2/

Gudynas, E. (2012). Debates sobre el desarrollo y sus alternativas. En M. L. (Comp.), Más Allá del Desarrollo (págs. 21-54). México: Fundación Rosa Luxemburg/Abya Yala.

López-Gatell, H. (2020). Conferencia 17 de julio. Conferencia de Prensa Covid-19. Chiapas, México: Secretaría de Salud.

Instituto Nacional de Estadística y Geografía. (2020). Cuéntame . Obtenido de Esperanza de vida: http://www.cuentame.org.mx/poblacion/esperanza.aspx?tema=P

Instituto Nacional de Salud Pública. (2020). Encuesta Nacional de Salud y Nutrición 2018. Presentación de resultados. Cuernavaca: INSP.

Instituto Nacional De Salud Pública, México. (2021). Gobierno de México. Obtenido de Publicidad de alimentos y bebidas: https://www.insp.mx/epppo/blog/2984-publicidad-alimentosbebidas.html 
DOI: 10.52191/rdojs.2021.207 La seguridad alimentaria, la malnutrición ... Págs.: 8-34

Martínez, M. (2019). Políticas sociales y participación en México : el caso del programa Comedores Comunitarios. Tluxtla Gutiérrez, Chiapas: Universidad de Cinecias y Artes de Chiapas.

Macari, Berumen, J., Calvillo, A. (2018). Publicidad dirigida a los niños: Una infancia enganchada a la obesidad. México: Alianza por la Salud Alimentaria.

Merton, R. (1992). Teoría y Estructuras Sociales. México: Fondo de Cultura Económica.

Notimex. (19 de Diciembre de 2018). Alianza por la salud alimentaria. Obtenido de Niños de 2 a 9 años comen más alimentos ultraprocesados, según estudio: https:// alianzasalud.org.mx/2018/12/ninos-de-2-a-9-anos-comen-mas-alimentos-ultraprocesadossegun-estudio/

Nozick, R. (2012). Anarquía, Estado y Útopia. México: Fondo de Cultura Económica.

Nussbaum, M. (2012). Crear capacidades. Propuesta para el desarrollo humano. España: Paidós.

Organización de las Naciones Unidas. (2021). Naciones Unidas. Obtenido de La Declaración Universal de Derechos Humanos: https://www.un.org/es/about-us/universal-declaration-ofhuman-rights

Organización de las Naciones Unidas. (2021). Naciones Unidas. Obtenido de Historia de la Declaración: https://www.un.org/es/about-us/udhr/history-of-the-declaration

Organización de las Naciones Unidas para la Alimentación y la Agricultura. (2011). La Seguridad Alimentaria: información para la toma de decisiones. Guía práctica. FAO.

Organización de las Naciones Unidas para la Alimentación y la Agricultura. (25 de Noviembre de 2019). FAO en México. Obtenido de México es donde más se comen alimentos ultraprocesados de América Latina y el Caribe: http://www.fao.org/mexico/noticias/detail-events/es/ c/1252734/\#: :text=El\%20consumo\%20de\%20alimentos\%20ultraprocesados,la\% 20Agricultura\%20(FAO)\%2C\%20el

Organización de las Naciones Unidas para la Alimentación y la Agricultura. (2020). Organic Agriculture . Obtenido de Preguntas frecuentes sobre agricultura: http://www.fao.org/organicag/oafaq/oa-faq5/es/

Organización Mundial de la Salud. (2016). Informe de la Comisión para acabar con la obesidad infantil. Ginebra: OMS. 
DOI: 10.52191/rdojs.2021.207 La seguridad alimentaria, la malnutrición ... Págs.: 8-34

Organización Mundial de la Salud. (2020). Sitio web mundial. Obtenido de Urbanización y salud: https://www.who.int/bulletin/volumes/88/4/10-010410/es/

Organización Panamericana de la Salud. (2015). Alimentos y bebidas ultraprocesados en América Latina: tendencias, efecto sobre la obesidad e implicaciones para las políticas <públicas. Washington, DC: OPS y OMS.

Organización Panamericana de la Salud. (2020). OPS. Obtenido de INDICADORES DE SALUD: Aspectos conceptuales y operativos (Sección 1): https://www.paho.org/hq/index.php? option=com_content\&view=article\&id=14401 :health-indicators-conceptual-and-operationalconsiderations-section-1\&ltemid=0\&showall=1\&lang=es

Preston, P. W. (1999). Una introducción a la teoría del desarrollo. México: Siglo XXI.

Programa de las Naciones Unidas para el Desarrollo. (2020). PNUD México. Obtenido de DesarroIlo Humano: https://www.mx.undp.org/content/mexico/es/home/ourwork/povertyreduction/ in_depth/desarrollo-humano.html

Rawls, J. (2002). La justicia como equidad. España: Paidós.

Secretaría de Agricultura y Desarrollo Rural. (2020). Gobierno de México. Obtenido de Canasta básica de alimentos: https://www.gob.mx/canastabasica

Secretaría de Educación Pública. (2017). LINEAMIENTOS PARA LA ORGANIZACIÓN Y EL FUNCIONAMIENTO DE LAS ESCUELAS DE TIEMPO COMPLETO. Educación Primaria. México: SEP.

Secretaría del Trabajo y Previsión Social. (17 de Diciembre de 2019). Gobierno de México. Obtenido de Con aumento de $20 \%$ al salario mínimo para 2020, México tiene las bases para crecer, afirma presidente López Obrador: https://www.gob.mx/stps/prensa/con-aumento-de-20al-salario-minimo-para-2020-mexico-tiene-las-bases-para-crecer-afirma-presidente-lopezobrador-230226?idiom=es\#: :text=Durante\%20su\%20intervenci\%C3\%B3n\%2C\%20la\% 20secretaria, $176.22 \% 20$ a\%20185.56\%20pesos\%20dia

Sen, A. (2000). Desarrollo y libertad. Barcelona: Planeta.

Sen, A. (2010). La idea de la justicia. España: Taurus.

UNICEF México. (2020). Salud y nutrición. Obtenido de https://www.unicef.org/mexico/salud-ynutrici\%C3\%B3n 